\title{
Tension across adherens junctions: when less is more
}

Editorial

\section{Kevin Kruse and Yulia A. Komarova}

The selective permeability of the endothelial monolayer is controlled by adherens junctions (AJs), which connect endothelial cells through adhesion of Vascular Endothelial (VE)- cadherin ectodomains (EC) in a strand-swap configuration, an anchorage of the conserved tryptophans Trp2 and Trp4 to a hydrophobic pocket of the opposing EC1 domain. This adhesion is a dynamic event characterized by the association $\left(k_{\text {on }}\right)$ and dissociation $\left(k_{\text {off }}\right)$ rates of the extracellular moiety of VEcadherin [1] (Figure 1). We have recently demonstrated that mutation of Trp2 and Trp4 significantly increases the VE-cadherin dissociation rate from AJs [1], suggesting that trans-dimerization "traps" VE-cadherin molecules at AJs. Hence, assembly of VE-cadherin adhesive bonds induces recruitment of VE-cadherin molecules to AJs by a diffusion trap mechanism to restrict permeability of the endothelial barrier to macromolecules.

The strength of VE-cadherin adhesive bonds, defined as the ability to sustain mechanical tension, requires attachment of the VE-cadherin adhesion complex to the actin cytoskeleton in a tension dependent manner [2]. In elegant studies by Buckley and colleagues, the interaction between the cadherin- $\beta / \alpha$-catenin complex and actin filaments was explained by a two state catch bond model in which the dissociation rate of F-actin from the cadherin complex decreases exponentially with respect to applied tension [2]. Using an optical trap-based assay, the authors showed that the cadherin-catenin complex forms stable bonds with F-actin filaments $<10 \mathrm{pN}$ whereas higher forces promote dissociation of F-actin from the complex [2]. These in vitro data and our most recent findings [1] suggest that assembly and strength of VE-cadherin adhesion is finely regulated by myosin-II-generated intracellular tension. This tension, depending on its distribution, nature, and magnitude, can favor VE-cadherin adhesion assembly or disassembly via modulation of the VE-cadherin dissociation rate (Figure 1).

Proteins of the adhesion complex not only provide a mechanism for cell-cell adhesion but also serve as sensors and transducers of mechanical tension. Sensors such as VE-cadherin and platelet endothelial cell adhesion molecule (PECAM-1) "perceive" intracellular forces $[3,4]$, whereas transducers such as $\alpha$-catenin and vinculin transmit force [5]. At mature junctions, $\alpha$-catenin undergoes rapid and reversible switching between tension states, suggesting the transient nature of traction forces [5].

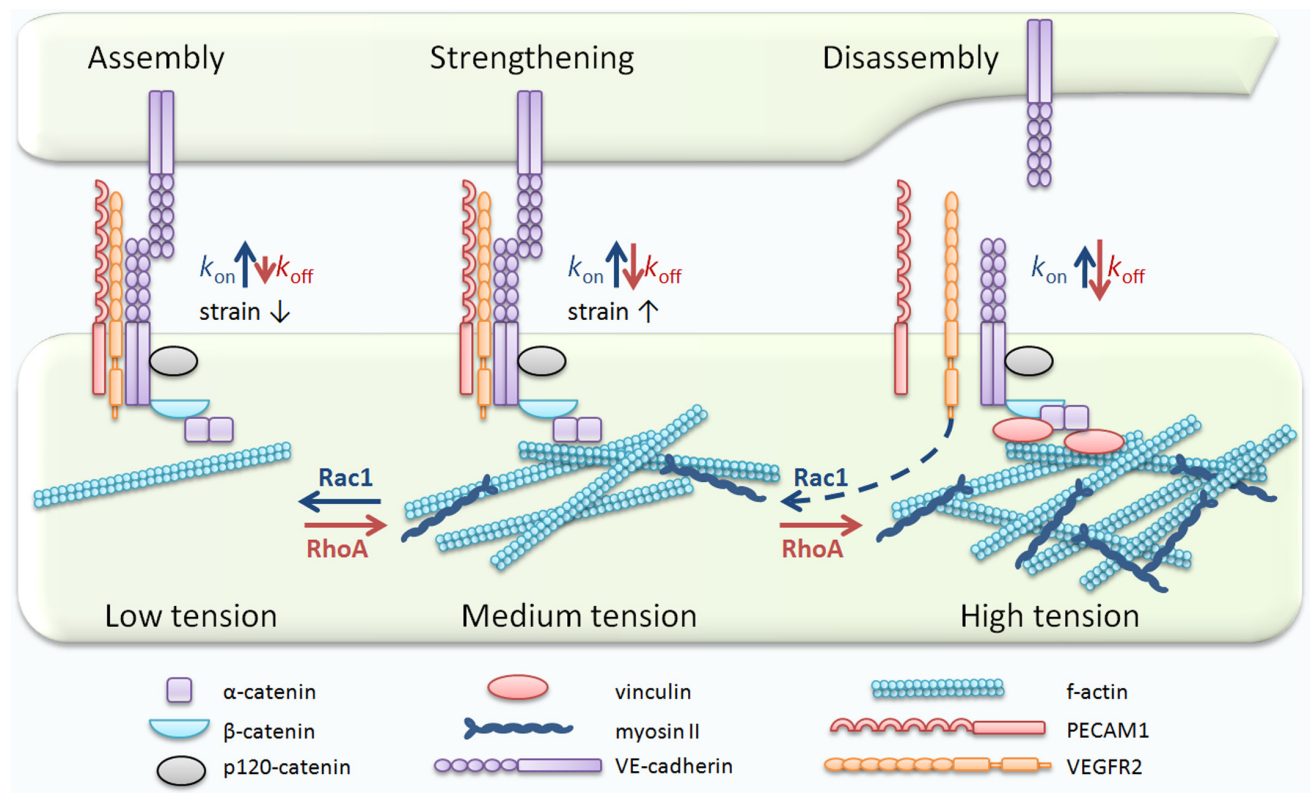

Figure 1: Relationship between tension and stability of VE-cadherin adhesions. VE-cadherin trans-dimerization promotes assembly of AJs whereas attachment of the VE-cadherin-catenin complex to the actin cytoskeleton increases the strength of AJs in a tension-dependent manner. Low tension promotes formation of stable bonds between the VE-cadherin-catenin complex and actin filaments whereas high tension disrupts VE-cadherin trans-interactions, inducing VE-cadherin dissociation from AJs. Tension also promotes conformational changes in $\alpha$-catenin, allowing for vinculin binding. Recruitment of vinculin to AJs induces re-distribution of tension while disruption of the mechanosensory complex with VEGFRs results in activation of Rac1, inducing reassembly of VE-cadherin adhesion. 
This might be a result of burst activation of intracellular signaling molecules such as the RhoGTPase Rac1, which we have shown to promote assembly of trans-dimers by altering RhoA and myosin-II activity and reducing tension across AJs [1]. Our model proposes that the relative activation levels of RhoA and Rac1 are essential drivers of assembly, stabilization, and remodeling of VE-cadherin adhesive bonds at mature AJs (Figure 1). RhoA promotes activation of myosin-II in a ROCK-dependent manner, which in turn generates intracellular forces needed for formation of stable bonds between actin filaments and the VE-cadherin complex. Rac1 counteracts these forces to promote VE-cadherin trans-dimerization by reducing the VE-cadherin dissociation rate and sequentially increasing VE-cadherin density at AJs. An increase in traction forces across VE-cadherin adhesions triggers prolonged conformational changes in $\alpha$-catenin leading to recruitment of vinculin to AJs $[5,6]$. This sequence of events might play a critical role in redistribution of localized forces. Vinculin can promote actin remodeling via Mena/VASP and Arp2/3-dependent pathways and tune assembly of AJs independently or in concert with Rac1 [6]. Hence, in our model, Rac1 promotes assembly of VE-cadherin adhesions whereas RhoA increases adhesion strength. This 'tug of war' between RhoA and Rac1 signaling at AJs is an important mechanism regulating the plasticity of VEcadherin adhesions and adaptation of endothelial cells to environmental cues.

The ability of cells to sense their environment is a critical factor in a variety of vascular processes such as adaptation of endothelial monolayers to fluid shear stress (FSS). VE-cadherin forms a junctional mechanosensory complex with PECAM-1 and vascular endothelial growth factor receptors (VEGFRs) [3, 4]. FSS induces ligand-independent activation of VEGFR signaling [4], resulting in decreased tension across VEcadherin adhesion [3], possibly through activation of Rac1. FSS also promotes association between PECAM1 and vimentin [3], an intermediate filament protein. The force-activated reorganization of intermediate filaments leads to redistribution of mechanical forces and prevents destabilization of VE-cadherin adhesions. This positive feedback mechanism plays a critical role in the adaptation of endothelial cells to environmental cues to help maintain barrier function and tissue homeostasis.

Yulia A. Komarova: Department of Pharmacology and The Center for Lung and Vascular Biology, University of Illinois at Chicago, College of Medicine, Chicago, IL, USA

Correspondence to: Yulia A. Komarova, email ykomarov@ vic.edu

Keywords: tension, adhesion, VE-cadherin, RhoGTPase, junction

Received: August 10, 2015

Published: September 01, 2015

\section{REFERENCES}

1. Daneshjou N, et al. J Cell Biol. 2015; 208: 23-32.

2. Buckley CD, et al. Science. 2014; 346: 1254211.

3. Conway DE, et al. Curr Biol. 2013; 23: 1024-30.

4. Coon BG, et al. J Cell Biol. 2015; 208: 975-86.

5. Kim TJ, et al. Curr Biol. 2015; 25: 218-24.

6. Leerberg JM, et al. Curr Biol. 2014; 24: 1689-99. 